\title{
Level Sets of the Takagi Function: Generic Level Sets
}

\author{
Jeffrey C. Lagarias ${ }^{1}$ \\ Dept. of Mathematics \\ University of Michigan \\ Ann Arbor, MI 48109-1043 \\ Zachary Maddock 2 \\ Dept. of Mathematics \\ Columbia University \\ New York, NY 10027
}

(July 26, 2011)

\begin{abstract}
The Takagi function $\tau:[0,1] \rightarrow[0,1]$ is a continuous non-differentiable function constructed by Takagi in 1903. This paper studies the level sets $L(y)=\{x: \tau(x)=y\}$ of the Takagi function $\tau(x)$. It shows that for a full Lebesgue measure set of ordinates $y$, these level sets are finite sets, but whose expected number of points is infinite. Complementing this, it shows that the set of ordinates $y$ whose level set has positive Hausdorff dimension is itself a set of full Hausdorff dimension 1 (but Lebesgue measure zero). Finally it shows that the level sets have a nontrivial Hausdorff dimension spectrum. The results are obtained using a notion of "local level set" introduced in a previous paper, along with a singular measure parameterizing such sets.
\end{abstract}

\section{Introduction}

The Takagi function $\tau(x)$ is a function defined on the unit interval $x \in[0,1]$ which was introduced by Takagi [23] in 1903 as an example of a continuous nondifferentiable function. It can be defined by

$$
\tau(x):=\sum_{n=0}^{\infty} \frac{\ll 2^{n} x \gg}{2^{n}}
$$

where $\ll x \gg$ is the distance from $x$ to the nearest integer. It has appeared in a wide variety of contexts in probability theory, number theory and analysis, including Bernoulli convolutions [14, p. 195], the distribution of binary digit sums ( ([22], [24], [8]), and as fractals and functions satisfying self-similar analogues of the Laplace equation ([25]).

This paper considers certain properties of the graph of the Takagi function

$$
\mathcal{G}(\tau):=\{(x, \tau(x)): 0 \leq x \leq 1\},
$$

\footnotetext{
${ }^{1}$ This author's work was supported by NSF Grants DMS-0500555 and DMS-0801029.

${ }^{2}$ This author's work was supported by the NSF through a Graduate Research Fellowship.
} 

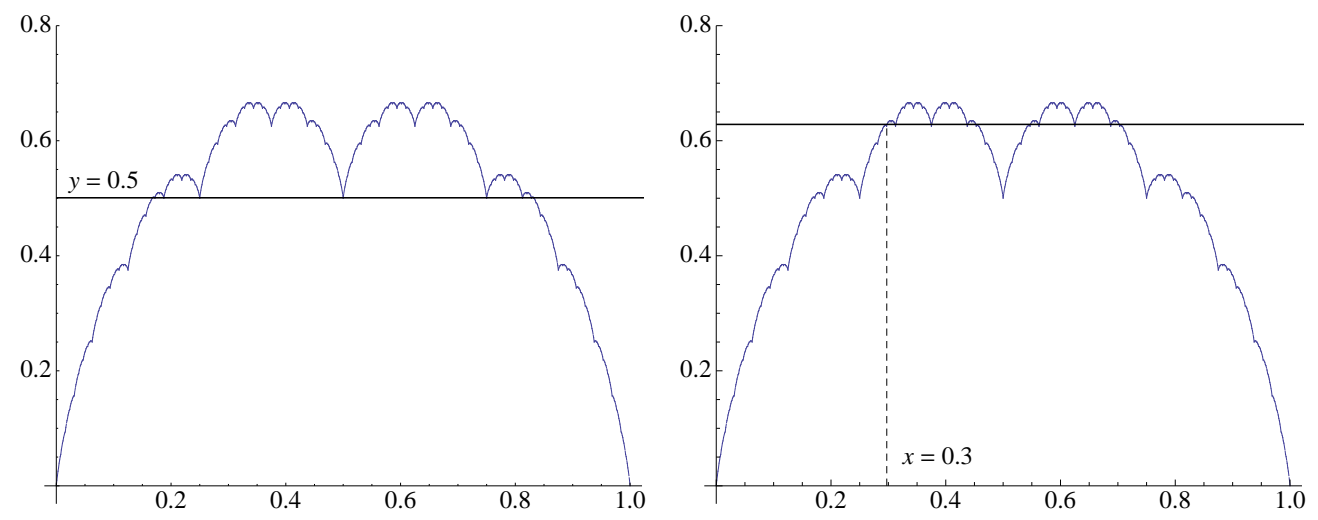

Figure 1: Ordinate level set $L(y)$ at $y=0.5$ and abscissa level set $L(\tau(x))$ at $x=0.3$.

which is pictured in Figure 1. It is well known that the values of the Takagi function satisfy $0 \leq$ $\tau(x) \leq \frac{2}{3}$. It is also known that this graph has Hausdorff dimension 1 in $\mathbb{R}^{2}$, see Mauldin and Williams [21, Theorem 7]). They add the remark that they do not know whether the 1-dimensional Hausdorff measure of this graph is finite or infinite ([21, p. 800]). Here we study the structure of the level sets of this graph. We make the following definition, which contains a special convention concerning dyadic rationals which simplifies theorem statements.

Definition 1.1. For $0 \leq y \leq \frac{2}{3}$ the (global) level set $L(y)$ at level $y$ is

$$
L(y):=\{x: \tau(x)=y, \quad 0 \leq x \leq 1\} .
$$

We make the convention that $x$ specifies a particular binary expansion; so each dyadic rational value $x=\frac{m}{2^{n}}$ in a level set will appear twice, labeled by each of its two possible binary expansions.

Level sets have a complicated and interesting structure, depending on the value of $y$. It is known that there are different levels $y$ where the level set $L(y)$ is finite, countably infinite, or uncountably infinite, respectively. Concerning the size of level sets, measured by Hausdorff dimension, in 1984 Baba [6] showed that the level set $L\left(\frac{2}{3}\right)$ has Hausdorff dimension $\frac{1}{2}$, so is uncountable. The second author showed ([20]) that the Hausdorff dimension of any level set is at most 0.699 and conjectured Baba's example achieves the largest possible dimension. Finally, relevant to our results here, in 2008 Buczolich [7] proved that, in the sense of Lebesgue measure, almost all level sets $L(y)$ are finite sets.

The object of this paper is to study properties of "generic" level sets of the Takagi function. The ordinate (y-axis) notion of genericity (in the Lebesgue measure sense) is to draw an ordinate $y$ at random using Lebesgue measure in $\left[0, \frac{2}{3}\right]$, and ask for properties of such level sets that hold for a full Lebesgue measure of such sets $L(y)$. The abscissa ( $x$-axis) notion of genericity is to draw a number $x$ at random in $[0,1]$ with respect to Lebesgue measure, and then to ask for properties of level sets $L(\tau(x))$ that hold for a full Lebesgue measure set of $x$. These two sampling methods of drawing level sets are pictured in Figure 1 above. We focus on ordinate notions of genericity, but also treat abscissa notions in order to obtain our results: we observe that these two notions of genericity give quite different answers concerning the structure of level sets.

A weaker notion of "generic set" is ask for properties of level sets that are generic in the Hausdorff dimension sense, which means to hold for a set of (full) Hausdorff dimension 1, in the appropriate variable. We consider this notion in both the abscissa sense and the ordinate sense. 


\subsection{Generic level sets: Results}

Our results are obtained using methods from our paper [19], which introduced the notion of "local level set" of the Takagi function. We summarize relevant results in Sect. 22 and 3. Local level sets are sets $L_{x}^{l o c}$ determined locally by combinatorial operations on the binary expansion of a real number $x$; they are closed sets and each level set is partitioned into a disjoint union of local level sets. The structure of local level sets $L_{x}^{l o c}$ is completely analyzable: they are either finite sets or Cantor sets. Information of the Hausdorff dimension of such sets can be deduced from properties of the binary expansion of $x$. In [19] we introduced a certain subset $\Omega^{L} \subset[0,1]$, called the deficient digit set, made up of the left endpoints of all local level sets; this set parameterizes all local level sets. We showed that $\Omega^{L}$ is a closed set of Lebesgue measure zero. We also introduced a new singular function $\mu_{S}$, the Takagi singular function, whose points of increase are supported on $\Omega^{L}$. This function was used to show that on almost all levels $y$ there are finitely many local level sets; however there is also a dense set of levels in $\left[0, \frac{2}{3}\right]$ which have infinitely many local level sets. In Theorem 3.2 below we introduce singular measure, the Takagi singular measure, associated to the Takagi singular function, which plays an important role in this paper.

Our first result concerns the cardinality of "generic" ordinate level sets in the Lebesgue measure sense.

Theorem 1.2. (Ordinate generic level sets) (1) For a full Lebesgue measure set of ordinate points $y \in\left[0, \frac{2}{3}\right]$ the level set $L(y)$ is a finite set.

(2) For a random level set $L(y)$ with level y drawn uniformly from $y \in\left[0, \frac{2}{3}\right]$, the expected number of elements in $L(y)$ is infinite.

We prove Theorem 1.2 in Sect. 4 and Sect. 5. This result is proved using explicit calculations of the Takagi singular measure of various subsets of $\Omega^{L}$ given in the fine decomposition of the deficient digit set $\Omega^{L}$ made in Sect. 4 below. These calculations make use of self-similarity properties of the Takagi singular measure.

Part (1) of this result was first proved in 2008 by Buczolich [7]. He proves the almost everywhere finiteness of level sets by a method that directly studies the graph of the Takagi function. His proof shows the graph $\mathcal{G}(\tau)=\mathcal{G}_{I} \cup \mathcal{G}_{R}$ (nonconstructively) partitions into an irregular 1-set $\mathcal{G}_{R}$ and a regular 1 -set $\mathcal{G}_{R}$, and that the irregular set $\mathcal{G}_{I}$ has $y$-projection of Lebesgue measure 0 and $x$-projection of full measure 1. Here an irregular 1-set or purely unrectifiable 1-set is a set in $\mathbb{R}^{2}$ of Hausdorff dimension 1 that intersects every continuously differentiable curve in a set of $\mathcal{H}^{1}$-measure zero. By Besicovich's theorem such a set has 1-dimensional projections of measure 0 in almost all directions, see Falconer [10, Theorem 6.1.3]. A regular 1-set is a set that can be covered by countably many rectifiable curves. In comparison our proof of Theorem 1.2 uses the Takagi singular measure, whose support lies in $\Omega^{L}$. The part of the graph $\mathcal{G}(\tau)$ that lies above $\Omega^{L}$ is covered by a single rectifiable curve, the flattened Takagi function described in [19, Sect. 5] (which is of bounded variation [19, Theorem 5.4]), so it should belong to the regular part $\mathcal{G}_{R}$ of Buczolich's partition.

We contrast Theorem 1.2 with what is known about "generic" abscissa level sets in the Lebesgue measure sense. In [19, Theorem 1.4] we showed that a "generic" local level set $L_{x}^{\text {loc }}$ obtained by drawing $x$ with the uniform distribution on $[0,1]$ (Lebesgue measure) is with probability one an uncountable set of Hausdorff dimension 0. An immediate consequence is: For a full Lebesgue measure set of abscissa points $x \in[0,1]$ the level set $L(\tau(x))$ is uncountable. Here we advance the following conjecture for abscissa level sets, concerning their Hausdorff dimension.

Conjecture 1.3. (Abscissa generic level sets) A full Lebesgue measure set of abscissa points $x \in[0,1]$ have level sets $L(\tau(x))$ that are uncountable and have Hausdorff dimension 0. 
We also prove generic results in the weaker Hausdorff dimension sense. Let $\operatorname{dim}_{H}(\Gamma)$ denote the Hausdorff dimension of a set $\Gamma$. Our next two results show that the set of level sets that are large in the sense of having positive Hausdorff dimension are themselves generic in the Hausdorff dimension sense. We prove results in both the abscissa generic case and the ordinate generic case.

Theorem 1.4. (Positive Hausdorff dimension abscissa level sets) Let $\Gamma_{H}^{a b s}$ be set of abscissas $x \in[0,1]$ such that the level set $L(\tau(x))$ has positive Hausdorff dimension, i.e.

$$
\Gamma_{H}^{a b s}:=\left\{x \in[0,1]: \operatorname{dim}_{H}(L(\tau(x)))>0\right\} .
$$

Then $\Gamma_{H}^{a b s}$ has full Hausdorff dimension, i.e.

$$
\operatorname{dim}_{H}\left(\Gamma_{H}^{a b s}\right)=1 .
$$

We do not obtain information about the Lebesgue measure of $\Gamma_{H}^{a b s}$. Conjecture 1.3 above is just the assertion that $\Gamma_{H}^{a b s}$ has Lebesgue measure 0 .

Theorem 1.4 is proved in Section 6, It is an immediate corollary of an analogous result for local level sets (Theorem 6.1), which proves Hausdorff dimension 1 even when one restricts to $x \in \Omega^{L}$. We define a family of Cantor sets $\Lambda_{2 r}$, contained in $\Gamma_{H}^{a b s} \cap \Omega^{L}$, where $r \geq 3$ is an integer parameter, and show that $\operatorname{dim}_{H}\left(\Lambda_{2 r}\right) \rightarrow 1$ as $r \rightarrow \infty$. Theorem 6.1 also shows: The deficient digit set $\Omega^{L}$ has Hausdorff dimension 1.

Theorem 1.5. (Positive Hausdorff dimension ordinate level sets) Let $\Gamma_{H}^{\text {ord }}$ be set of ordinates $y \in\left[0, \frac{2}{3}\right]$ such that the Takagi function level set $L(y)$ has positive Hausdorff dimension, i.e.

$$
\Gamma_{H}^{o r d}:=\left\{y: \operatorname{dim}_{H}(L(y))>0\right\} .
$$

Then $\Gamma_{H}^{o r d}$ has full Hausdorff dimension, i.e.

$$
\operatorname{dim}_{H}\left(\Gamma_{H}^{o r d}\right)=1 .
$$

Here we know that the set $\Gamma_{H}^{o r d}$ has Lebesgue measure 0 , a result that follows from Theorem 1.2 .

Theorem 1.5 is proved in Sect. 7. We show that the Takagi function $\tau(x)$ restricted to each Cantor set $\Lambda_{2 r}$ in Sect. 6is strictly increasing and is a bi-Lipschitz map. The result follows since bi-Lipschitz maps preserve Hausdorff dimension.

\subsection{Dimension Spectrum of Takagi Level Sets}

Our results establish that level sets of the Takagi function exhibit a nontrivial dimension spectrum. We define the dimension spectrum function for Takagi level sets to be the function

$$
f_{\tau}(\alpha):=\operatorname{dim}_{H}\left\{y: \operatorname{dim}_{H}(L(y)) \geq \alpha\right\} .
$$

By definition this function is a nonincreasing function of $\alpha$. The second author conjectured [20], and de Amo et al. [5] proved, that there are no level sets having Hausdorff dimension exceeding $\frac{1}{2}$, which gives

$$
f_{\tau}(\alpha)=0 \text { for } \alpha>\frac{1}{2}
$$


We trivially have $f_{\tau}(0)=1$ and Theorem 1.5 of this paper establishes that $\lim _{\alpha \rightarrow 0^{+}} f_{\tau}(\alpha)=1$, by showing that for all sufficiently large integers $r$,

$$
f_{\tau}\left(\frac{1}{2 r}\right) \geq 1-\frac{2 \log r}{2 r} .
$$

By monotonicity of this function we have $f_{\tau}(\alpha)>0$ holding on some interval $\left[0, \alpha_{0}\right)$ of positive length. It seems reasonable to expect that $f_{\tau}(\alpha)>0$ for $0 \leq \alpha<\frac{1}{2}$. Finally, we note that the assertion that $f_{\tau}(\alpha)<1$ holds for each $\alpha>0$ would imply the truth of Conjecture 1.3 .

One can also study analogous questions for local level sets. We define the local level set dimension spectrum function by

$$
f_{\tau}^{*}(\alpha):=\operatorname{dim}_{H}\left(\left\{x \in \Omega^{L}: \operatorname{dim}_{H}\left(L_{x}^{l o c}\right) \geq \alpha\right\}\right) .
$$

This function is not directly comparable with $f_{\tau}(\alpha)$ because it samples abscissa points rather than ordinate points. The nonexistence of level sets of Hausdorff dimension exceeding $\frac{1}{2}$ yields

$$
f_{\tau}^{*}(\alpha)=0 \text { for } \alpha>\frac{1}{2} \text {. }
$$

Theorem 6.1 of this paper establishes that $f_{\tau}^{*}(0)=\lim _{\alpha \rightarrow 0^{+}} f_{\tau}^{*}(\alpha)=1$, by showing that, for all large enough integers $r$,

$$
f_{\tau}^{*}\left(\frac{1}{2 r}\right)>1-\frac{2 \log r}{r}
$$

Again it follows that $f_{\tau}^{*}(x)>0$ on some interval $\left[0, \alpha_{1}\right)$ of positive length.

The notion of multifractal formalism (or thermodynamic formalism) has been introduced in connection with the Hölder spectrum of points of a nonsmooth function having given Hölder exponent; see Jaffard [15], [16] for discussion and many references. It is predicted (under suitable hypotheses) that the Hölder spectrum dimension $f(\alpha)$ of a given function is a real-analytic function of $\alpha$ over a certain range, and also exhibits a convexity property over this range. The multifractal formalism further relates local properties of the function (local Hölder exponents) to global smoothness properties of the function, invoving a Legendre transform of the function $f(\alpha)$. Our dimension functions above encode rather different properties of the Takagi function-size of its level sets- but in a certain sense still sample local properties of this function. Therefore it seems reasonable to ask whether the associated dimension functions $f_{\tau}(\alpha)$ and $f_{\tau}^{*}(\alpha)$ might have similar analytic and convexity properties.

\subsection{Discussion}

There has been much prior study of the non-differentiable structure of the Takagi function and related functions according to various measures. See the papers of Allaart and Kawamura ([3], [4]), and references therein. We also mention the survey paper of the first author [18]. Level sets study only one particular aspect of the nondifferentiability of this function.

An interesting feature of the Takagi function is that the cardinality of "generic" abscissa and "generic" ordinate level sets in the Lebesgue measure sense differ drastically. This difference can occur because sampling a point $x$ on the abscissa favors picking level sets which are "large." It is a manifestation of the non-differentiable nature of the Takagi function. This difference indicates that the Takagi function must (in some sense) have "infinite slope" over part of its domain. In particular $\tau(x)$ is not a function of bounded variation.

We raise as open problems the determination of the dimension functions $f_{\tau}(\alpha)$ and $f_{\tau}^{*}(\alpha)$ for $0 \leq \alpha \leq 1$ and of deciding whether properties predicted by the thermodynamic formalism hold. It 
might conceivably be true that $f_{\tau}(\alpha)=f_{\tau}^{*}(\alpha)$ holds for $0 \leq \alpha \leq 1$; the above discussion shows it holds for $\alpha \geq \frac{1}{2}$.

Some further improvements of the results on level sets appear in Allaart [1], [2]. In particular [2] obtains results on the detailed distribution of level sets having a given finite number of elements.

Acknowledgments. The first author thanks D. E. Knuth for raising interesting questions about the Takagi function (see [17, Problem 82, p. 103].) We thank Pieter Allaart for allowing use to include his substantially simplified proof of Theorem[7.1, and for bringing the work of of Buczolich [7] to our attention. We thank the reviewer for helpful comments.

\section{Preliminaries: Properties of the Takagi Function}

\subsection{Functional Equations}

We first recall two functional equations satisfied by the Takagi function [19, Lemma 2.2].

Lemma 2.1. (Takagi functional equations) The Takagi function satisfies two functional equations, valid for $0 \leq x \leq 1$, the reflection equation

$$
\tau(x)=\tau(1-x)
$$

and the dyadic self-similarity equation

$$
2 \tau\left(\frac{x}{2}\right)=\tau(x)+x
$$

We next formulate a local self -similarity property of the graph of the Takagi function. To describe it we require some functions determined by the binary expansion of $x$.

Definition 2.2. Let $x$ denote a binary expansion

$$
x:=\sum_{j=1}^{\infty} \frac{b_{j}}{2^{j}}=0 . b_{1} b_{2} b_{3} \ldots,
$$

with each $b_{j} \in\{0,1\}$. For each $j \geq 1$ we define the following integer-valued functions.

(1) The digit sum function $N_{j}^{1}(x)$ is

$$
N_{j}^{1}(x):=b_{1}+b_{2}+\cdots+b_{j} .
$$

We also set

$$
N_{j}^{0}(x):=j-N_{j}^{1}(x) .
$$

These functions count the number of 1's (resp. 0's) in the first $j$ binary digits of $x$.

(2) The deficient digit function $D_{j}(x)$ is given by

$$
D_{j}(x):=N_{j}^{0}(x)-N_{j}^{1}(x)=j-2 N_{j}^{1}(x)=j-2\left(b_{1}+b_{2}+\cdots+b_{j}\right) .
$$

The function $D_{j}(x)$ counts the excess of binary digits $b_{k}=0$ over those with $b_{k}=1$ in the first $j$ digits, i.e. it is positive if there are more 0's than 1's. (Note that dyadic rationals have two different binary expansions, and the functions $N_{j}^{0}(x), N_{j}^{1}(x), D_{j}(x)$ depend on the choice of expansion.) 
The local self-similarity property of the Takagi function graph is as follows ([19, Lemma 2.5(2)]).

Lemma 2.3. (Takagi self-similarity)

Let $x_{0}=0 . b_{1} b_{2} \ldots b_{n} 0^{\infty}=\frac{k}{2^{n}}$ be a dyadic rational, and parameterize the interval $\left[\frac{k}{2^{n}}, \frac{k+1}{2^{n}}\right]$ as $x=x_{0}+\frac{w}{2^{n}}$ for $0 \leq w \leq 1$. Then there holds

$$
\tau(x)=\tau\left(x_{0}\right)+\frac{1}{2^{n}}\left(\tau(w)+D_{n}\left(x_{0}\right) w\right) .
$$

That is, on the dyadic interval $\left[\frac{k}{2^{n}}, \frac{k+1}{2^{n}}\right]$ the graph of the function $\tau(x)$ is a miniature version of its full graph, vertically shifted by $\tau\left(x_{0}\right)$, shrunk by a factor $\frac{1}{2^{n}}$, and tilted by an additive linear factor $\frac{1}{2^{n}} D_{n}\left(x_{0}\right) w$.

In particular, for the case of balanced dyadic rationals, which are ones with $D_{n}\left(x_{0}\right)=0$ (necessarily $n=2 m$ is even), (2.7) simplifies to

$$
\tau(x)=\tau\left(x_{0}\right)+\frac{\tau(w)}{2^{n}},
$$

which comprises only a vertical shift and shrinking of the Takagi function.

\subsection{Local level sets}

The notion of local level set $L_{x}^{l o c}$ is attached to the binary expansion of an abscissa point $x \in[0,1]$. We show that certain combinatorial flipping operations applied to the binary expansion of $x$ yield new points $x^{\prime}$ in the same level set. The totality of points reachable from $x$ by these combinatorial operations will comprise the local level set $L_{x}^{\text {loc }}$ associated to $x$.

Let a binary expansion of $x \in[0,1]$ be given:

$$
x:=\sum_{j=1}^{\infty} \frac{b_{j}}{2^{j}}=0 . b_{1} b_{2} b_{3} \ldots, \quad \text { each } b_{j} \in\{0,1\} .
$$

The flip operation (or complementing operation) on a single binary digit $b$ is

$$
\bar{b}:=1-b .
$$

We associate to any binary expansion $x$ the sequence of digit positions $j$ at which tie-values of the deficient digit function $D_{j}(x)=0$ occur, which we call balance points; note that all such $j$ are even. The balance-set $Z(x)$ associated to $x$ is denoted

$$
Z(x):=\left\{c_{k}: D_{c_{k}}(x)=0\right\} .
$$

where we define $c_{0}=c_{0}(x)=0$ and set $c_{0}(x)<c_{1}(x)<c_{2}(x)<\ldots$. This sequence of tie-values may be finite or infinite. If it is finite, ending in $c_{n}(x)$, we make the convention to adjoin a final "balance point" $c_{n+1}(x)=+\infty$. We call a "block" an indexed set of digits between two consecutive balance points,

$$
B_{k}(x):=\left\{b_{j}: c_{k}(x)<j \leq c_{k+1}(x)\right\},
$$

which includes the second balance point but not the first. We define an equivalence relation on blocks, written $B_{k}(x) \sim B_{k^{\prime}}\left(x^{\prime}\right)$ to mean the block endpoints agree $\left(c_{k}(x)=c_{k^{\prime}}\left(x^{\prime}\right)\right.$ and $c_{k+1}(x)=$ 
$\left.c_{k^{\prime}+1}\left(x^{\prime}\right)\right)$ and either $B_{k}(x)=B_{k^{\prime}}\left(x^{\prime}\right)$ or $B_{k}(x)=\bar{B}_{k^{\prime}}\left(x^{\prime}\right)$, where the bar operation flips all the digits in the block, i.e.

$$
b_{j} \mapsto \bar{b}_{j}:=1-b_{j}, \quad c_{k}<j \leq c_{k+1} .
$$

Finally, we define the equivalence relation $x \sim x^{\prime}$ on two binary expansions to mean that they have identical balance-sets $Z(x) \equiv Z\left(x^{\prime}\right)$, and furthermore every block $B_{k}(x) \sim B_{k}\left(x^{\prime}\right)$ for $k \geq 0$. Note that $x \sim 1-x$; this corresponds to a flipping operation being applied to every binary digit. In [19] we showed that the equivalence relation $x \sim x^{\prime}$ implies that $\tau(x)=\tau\left(x^{\prime}\right)$, so that $x$ and $x^{\prime}$ are in the same level set of the Takagi function.

Definition 2.4. The local level set $L_{x}^{\text {loc }}$ associated to $x$ is the set of equivalent points,

$$
L_{x}^{l o c}:=\left\{x^{\prime}: x^{\prime} \sim x\right\} .
$$

We use again the convention that $x$ and $x^{\prime}$ denote binary expansions, so that dyadic rationals require special treatment.

We recall some basic properties of local level sets ([19, Theorem 3.1, Corollary 3.2]).

1. Local level sets $L_{x}^{l o c}$ are closed sets. Two local level sets either coincide or are disjoint.

2. Each local level set $L_{x}^{l o c}$ is contained in a level set: $L_{x}^{l o c} \subseteq L(\tau(x))$. That is, if $x_{1} \sim x_{2}$ then $\tau\left(x_{1}\right)=\tau\left(x_{2}\right)$.

3. Each level set $L(y)$ partitions into local level sets

$$
L(y)=\bigcup_{\substack{x \in \Omega^{L} \\ \tau(x)=y}} L_{x}^{l o c}
$$

Here $\Omega^{L}$ denotes the collection of leftmost endpoints of all local level sets.

4. A local level set $L_{x}^{l o c}$ is a finite set if the balance-set $Z(x)$ is finite; otherwise it is an uncountable perfect set (Cantor set).

\subsection{Deficient digit set $\Omega^{L}$}

In [19] we studied the set of leftmost endpoints $\Omega^{L}$ of local level sets.

Definition 2.5. The deficient digit set $\Omega^{L}$ consists of all $x$ such that

$$
\Omega^{L}:=\left\{x=\sum_{j=1}^{\infty} \frac{b_{j}}{2^{j}}: D_{j}(x) \geq 0 \text { for all } j \geq 1\right\} .
$$

The deficient digit set is a Cantor-type set obtained by removing a certain countable collection of open intervals from the unit interval, which we describe using the following definitions.

Definition 2.6. (1) The breakpoint set $\mathcal{B}^{\prime}$ consists of $B_{\emptyset}{ }^{\prime}=0$ together with the collection of all balanced dyadic rationals in $\Omega^{L}$. These are all $B^{\prime}=\frac{n}{2^{2 m}}$ that have binary expansions of the form

$$
B^{\prime}=0 . b_{1} b_{2} \ldots b_{2 m-1} b_{2 m} 0^{\infty} \text { for some } m \geq 1
$$


that satisfy the condition

$$
D_{j}\left(B^{\prime}\right) \geq 0 \text { for } 1 \leq j \leq 2 m-1, \quad \text { and } \quad D_{2 m}\left(B^{\prime}\right)=0,
$$

This condition implies $b_{2 m}=1$.

(2) The small breakpoint set $\mathcal{B}$ is the subset of the breakpoint set $\mathcal{B}^{\prime}$ consisting of $B_{\emptyset}=0$ plus all dyadic rationals in $\mathcal{B}^{\prime}$ that satisfy the extra condition that the last two binary digits $b_{2 m-1}=b_{2 m}=1$.

In [19] we used the small breakpoint set $\mathcal{B}$ to label the intervals removed from $[0,1]$ to create the deficient digit set $\Omega^{L}$.

Definition 2.7. For each dyadic rational $B=0 . b_{1} b_{2} \ldots b_{l} 01^{k}, k \geq 2$ in the small breakpoint set $\mathcal{B}$ $\left(B \neq B_{\emptyset}\right)$ associate the open interval $I_{B}:=\left(x(B)^{-}, x(B)^{+}\right)$having endpoints

$$
\begin{aligned}
& x(B)^{-}:=0 . b_{1} b_{2} \ldots b_{l} 01^{k}(01)^{\infty} \\
& x(B)^{+}:=0 . b_{1} b_{2} \ldots b_{l} 10^{k}(00)^{\infty},
\end{aligned}
$$

necessarily with $k \geq 2$. We also set $I_{B_{\emptyset}}=\left(\frac{1}{3}, 1\right)$.

The following result gives properties of the deficient digit set $\Omega^{L}$ ([19, Theorem 4.6].)

Theorem 2.8. (Properties of Deficient Digit Set)

(1) The deficient digit set $\Omega^{L}$ comprises the set of leftmost endpoints of all local level sets. It satisfies $\Omega^{L} \subset\left[0, \frac{1}{3}\right]$.

(2) The deficient digit sum set $\Omega^{L}$ is a closed, perfect set (Cantor set). It is given by

$$
\Omega^{L}=[0,1) \backslash \bigcup_{B \in \mathcal{B}} I_{B}
$$

where the omitted open intervals $I_{B}$, for $B$ in the small breakpoint set, have right endpoint a dyadic rational and left endpoint a rational number with denominator $3 \cdot 2^{k}$ for some $k \geq 1$.

(3) The deficient digit set $\Omega^{L}$ has Lebesgue measure zero.

In [19, Lemma 4.5] it is shown that the value of the endpoints of the removed intervals satisfies

$$
x_{B}^{+}-x_{B}^{-}=\tau\left(x(B)^{-}\right)-\tau\left(x(B)^{+}\right)=\frac{1}{2^{k+l} \cdot 3},
$$

so that linear interpolation of a function across the a removed interval always has slope -1 .

\subsection{Takagi function on deficient digit set}

In [19, Theorem 4.8] we proved that the Takagi function is nondecreasing on the set $\frac{1}{2} \Omega^{L}$. Note that

$$
\frac{1}{2} \Omega^{L}:=\left\{\frac{1}{2} x: x \in \Omega^{L}\right\}=\left\{x \in[0,1]: D_{j}(x)>0 \text { for all } j \geq 1\right\},
$$

which shows that $\frac{1}{2} \Omega^{L} \subset \Omega^{L}$.

Theorem 2.9. (1) The Takagi function is nondecreasing on the set $\frac{1}{2} \Omega^{L}$.

(2) The Takagi function is strictly increasing on $\frac{1}{2} \Omega^{L}$ away from a countable set of points, which are a subset of those rationals having binary expansions ending in $0^{\infty}$ or $(01)^{\infty}$. For each level $y$ the equation $y=\tau(x)$ has at most two solutions with $x \in \frac{1}{2} \Omega^{L}$. Thus if $x_{1}<x_{2}<x_{3}$ all belong to $\frac{1}{2} \Omega^{L}$ then $\tau\left(x_{3}\right)>\tau\left(x_{1}\right)$.

This result will be used in establishing the bi-Lipschitz property appearing in Theorem 7.1 


\section{Takagi Singular Measure}

A main ingredient used in this paper will be a singular measure, which is the weak derivative of the Takagi singular function constructed in [19]. We summarize the basic facts on the Takagi singular function, taken from [19, Theorems 1.5 ]. It is pictured in Figure 2 below.

Theorem 3.1. (Takagi singular function) The function $\tau^{S}(x)$ defined by $\tau^{S}(x)=\tau(x)+x$ for $x \in \Omega^{L}$ is a nondecreasing function on $\Omega^{L}$. Define its extension to all $x \in[0,1]$ by

$$
\tau^{S}(x):=\sup \left\{\tau^{S}\left(x_{1}\right): x_{1} \leq x \text { with } x_{1} \in \Omega^{L}\right\} .
$$

Then the function $\tau^{S}(x)$ is a monotone singular function. That is, it is a nondecreasing continuous function having $\tau^{S}(0)=0, \tau^{S}(1)=1$, which has derivative zero at (Lebesgue) almost all points of $[0,1]$. The closure of the set of points of increase of $\tau^{S}(x)$ is the deficient digit set $\Omega^{L}$.

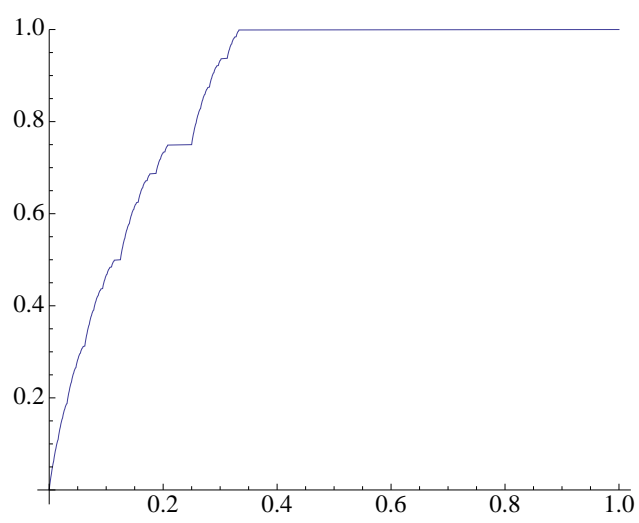

Figure 2: Graph of the singular Takagi function.

The weak derivative of the Takagi singular function is a Radon measure $\mu_{S}$ that we call the Takagi singular measure, and it has the following properties.

Theorem 3.2. (Takagi singular measure) The Takagi singular function $\tau^{S}(x)$ is the definite integral of a nonnegative Radon measure $\mu_{S}$, i.e. $\tau^{S}(x)=\int_{0}^{x} d \mu_{S}$. The measure $\mu_{S}$ has support equal to the deficient digit set $\Omega^{L}$, so that

$$
\int_{0}^{1} d \mu_{S}=\int_{\Omega^{L}} d \mu_{S}=1
$$

Thus it is singular continuous with respect to Lebesgue measure. For every Borel set $K$ in $[0,1]$,

$$
\mu_{S}(K)=\operatorname{meas}\left(\tau^{S}(K)\right)
$$

where meas $(\cdot)$ denotes Lebesgue measure.

Proof. By Theorem 3.1, the function $\tau^{S}(x)$ is monotone and bounded, hence of bounded (pointwise) variation. It follows that its distributional derivative is a finite Radon measure, call it $\mu_{S}$. It is necessarily nonnegative since $\tau^{S}(x)$ is nondecreasing (cf. [9, Theorem 1, Sect. 5.1]).

The support of a measure $\mu$ on the real line is the closure of the set of points of increase of $\mu$, which is $\Omega^{L}$ by Theorem 3.1. Since meas $\left(\Omega^{L}\right)=0$ it is a singular measure, and it is singular continuous 
because the integral $\tau^{S}(x):=\int_{0}^{x} d \mu_{S}$ is a continuous function of $x$. Finally, since $[0,1]$ is compact, any Radon measure (inner regular measure) on $[0,1]$ is also outer regular ([13, Theorem E, Sect. 52]). Outer regularity means that for any Borel set $K, \mu_{S}(K)=\inf _{K \subset U}\left\{\mu_{S}(U): U\right.$ an open set $\}$. We know that the Takagi singular measure of an open interval $U=(0, t)$ is

$$
\mu_{S}(U):=\int_{0}^{t} d \mu_{S}(x)=\tau^{S}(t)-\tau^{S}(0)=\tau^{S}(t)=\operatorname{meas}\left(\left(\tau^{S}(0), \tau^{S}(t)\right)\right)=\operatorname{meas}\left(\tau^{S}(U)\right) .
$$

where meas denotes Lebesgue measure. Outer regularity of both $\mu_{S}$ and of Lebesgue measure now implies that the equality holds on every Borel set $K$, proving (3.20).

\section{Structure of Takagi Singular Measure}

We now compute values of the Takagi singular measure on various subsets of $\Omega^{L}$ In $\$ 4.1$ we define a fine partition of $\Omega^{L}$ and in the remaining subsections we compute the singular measures of all sets in this partition.

\subsection{Fine partition of deficient digit set}

We partition the set $\Omega^{L}$ of left endpoints of local level sets into finer pieces, as follows:

$$
\Omega^{L}=\Omega_{\infty}^{L} \bigcup \Omega_{f i n}^{L}
$$

in which

$$
\Omega_{\infty}^{L}:=\left\{x \in \Omega^{L}: D_{j}(x)=0 \text { for infinitely many } j \geq 1\right\} .
$$

and $\Omega_{f i n}^{L}$ is its complement,

$$
\Omega_{f i n}^{L}:=\left\{x \in \Omega^{L}: D_{j}(x) \geq 1 \text { for all sufficiently large } j\right\} .
$$

The latter set can be further partitioned into subsets labelled by elements $B^{\prime}$ in the breakpoint set $\mathcal{B}^{\prime}$ consisting of all dyadic rationals $B^{\prime}=0 . b_{1} b_{2} . . b_{2 m}=\frac{k}{2^{2 m}}$ such that all $D_{j}\left(B^{\prime}\right) \geq 0$ and $D_{2 m}\left(B^{\prime}\right)=0$. To each $B^{\prime} \in \mathcal{B}^{\prime}$ we associate the set

$$
\Omega^{L}\left(B^{\prime}\right):=\left\{x=B^{\prime}+\frac{x^{\prime}}{2^{2 m}}: x^{\prime} \in \frac{1}{2} \Omega^{L}\right\} .
$$

In particular for $m=0$ we have one set $B^{\prime}=B_{0}=0$ with $\Omega^{L}\left(B_{0}\right)=\frac{1}{2} \Omega^{L}$.

Lemma 4.1. (Fine Partition of Deficient Digit Set) The set $\Omega_{\text {fin }}^{L}$ has a partition

$$
\Omega_{f i n}^{L}=\bigcup_{B^{\prime} \in \mathcal{B}^{\prime}} \Omega^{L}\left(B^{\prime}\right)
$$

with union over the breakpoint set $\mathcal{B}^{\prime}$. Each set $\Omega^{L}\left(B^{\prime}\right)$ is a closed set.

Proof. Elements $x \in \Omega^{L}\left(B^{\prime}\right)$ have $D_{j}(x) \geq 0$ for all $j \geq 1, D_{2 m}(x)=D_{2 m}\left(B^{\prime}\right)=0$, and $D_{j}(x)>0$ for $j \geq 2 m+1$. In particular $\Omega^{\bar{L}}\left(B^{\prime}\right) \subset \Omega_{\text {fin }}^{L}$. The sets are disjoint for different $B^{\prime}$ because the value $2 m$ is uniquely determined for each element of the set $\Omega^{L}\left(B^{\prime}\right)$, and this determines the initial digits $B^{\prime}$ uniquely. Finally we see that each element $x \in \Omega_{f i n}^{L}$ has associated to it a unique maximal value $2 m$ of $j$ such that $D_{j}(x)=0,(j$ is necessarily even) and this assigns it to a particular $\Omega^{L}\left(B^{\prime}\right)$. 


\subsection{Singular measure mass calculations}

The Takagi singular measure $d \mu_{S}$ is not translation-invariant. However in Theorem 4.2 below we are able to use its self-similarity properties to compute the $\mu_{S}$-measure of certain sets inside $\Omega^{L}$, namely the sets $\Omega_{\infty}^{L}$ and the sets $\Omega^{L}\left(B^{\prime}\right)$ in the fine partition of $\Omega^{L}$ in Lemma 4.1. where $B^{\prime}$ runs over the set of balanced dyadic rationals that belong to $\Omega^{L}$.

Theorem 4.2. For each balanced dyadic rational $B^{\prime}=0 . b_{1} b_{2} \ldots b_{2 m}=\frac{k}{2^{2 m}}$ in the deficient digit set $\Omega^{L}$ the fine partition set $\Omega^{L}\left(B^{\prime}\right)$ is a closed set, and its Takagi singular measure is

$$
\mu_{S}\left(\Omega^{L}\left(B^{\prime}\right)\right):=\int_{\Omega^{L}\left(B^{\prime}\right)} d \mu_{S}=\frac{1}{2^{2 m+1}} .
$$

Proof. We already know that $\mu_{S}\left(\Omega^{L}\right)=1$ via Theorem 3.2 .

Claim 1. The Takagi singular measure mass of $\frac{1}{2} \Omega^{L}$ is given by

$$
\mu_{S}\left(\Omega^{L}\left(B_{0}\right)\right):=\mu_{S}\left(\frac{1}{2} \Omega^{L}\right)=\frac{1}{2} \mu_{S}\left(\Omega^{L}\right)=\frac{1}{2} .
$$

To prove the claim, we use the self-similarity relation in Lemma 2.1.(1),

$$
2 \tau\left(\frac{1}{2} x\right)=x+\tau(x), \quad \text { for } 0 \leq x \leq 1 .
$$

It $x \in \Omega^{L}$ then $\tau(x)=\tau^{L}(x)$ so that we obtain

$$
2 \tau\left(\frac{1}{2} x\right)=x+\tau(x)=\tau^{S}(x) .
$$

Thus if $x_{1}<x_{2}$ with both $x_{i} \in \Omega^{L}$, then

$$
\begin{aligned}
\int_{\frac{1}{2} x_{1}}^{\frac{1}{2} x_{2}} \mu_{S} & =\tau^{S}\left(\frac{1}{2} x_{2}\right)-\tau^{S}\left(\frac{1}{2} x_{1}\right) \\
& =\left(\tau\left(\frac{1}{2} x_{2}\right)+\frac{1}{2} x_{2}\right)-\left(\tau\left(\frac{1}{2} x_{1}\right)+\frac{1}{2} x_{1}\right) \\
& =\frac{1}{2}\left(\tau^{S}\left(x_{2}\right)-\tau^{S}\left(x_{1}\right)\right)+\frac{1}{2}\left(x_{2}-x_{1}\right) \\
& =\frac{1}{2} \int_{x_{1}}^{x_{2}} \mu_{S}+\frac{1}{2}\left(x_{2}-x_{1}\right) .
\end{aligned}
$$

We may rewrite this as

$$
\left|\int_{\frac{1}{2} x_{1}}^{\frac{1}{2} x_{2}} \mu_{S}-\frac{1}{2} \int_{x_{1}}^{x_{2}} \mu_{S}\right| \leq \frac{1}{2} \operatorname{meas}\left(\left[x_{1}, x_{2}\right]\right)
$$

where the last term denotes the Lebesgue measure of the interval $\left[x_{1}, x_{2}\right]$.

Now by 2.17 for each $m \geq 1$ we obtain a covering of $\Omega^{L}$ using

$$
\Omega^{L} \subset \mathcal{P}_{2 m}:=[0,1) \backslash \bigcup_{\substack{B \in \mathcal{B} \\|B| \leq 2 m}} I_{B}
$$


in which we remove only a finite number of the "flattened" open intervals $I_{B}$ corresponding to those $B \in \mathcal{B}$ (the small breakpoint set) having dyadic length at most $2 m$. The set $\mathcal{P}_{2 m}$ is a closed set comprised of a finite number of intervals, $\left[x_{j}, x_{j}^{\prime}\right]$, say, having both endpoints $x_{j}, x_{j}^{\prime} \in \Omega^{L}$. Adding up the relations 4.10) over these intervals yields

$$
\left|\int_{\frac{1}{2} \mathcal{P}_{2 m}} \mu_{S}-\frac{1}{2} \int_{\mathcal{P}_{2 m}} \mu_{S}\right| \leq \frac{1}{2} \operatorname{meas}\left(\mathcal{P}_{2 m}\right),
$$

in which meas $\left(\mathcal{P}_{2 m}\right)$ denotes the Lebesgue measure of the set $\mathcal{P}_{2 m}$. Next we note that the $\mathcal{P}_{2 m}$ form a nested family $\mathcal{P}_{2} \supset \mathcal{P}_{4} \supset \mathcal{P}_{6} \supset \cdots$ of closed sets, with

$$
\Omega^{L}=\bigcap_{m=1}^{\infty} \mathcal{P}_{2 m}
$$

Since these sets are Borel measurable and $\mu_{S}$ is outer regular we have

$$
\begin{aligned}
\lim _{m \rightarrow \infty} \int_{\mathcal{P}_{2 m}} \mu_{S} & =\int_{\Omega^{L}} \mu_{S}, \\
\lim _{m \rightarrow \infty} \int_{\frac{1}{2} \mathcal{P}_{2 m}} \mu_{S} & =\int_{\frac{1}{2} \Omega^{L}} \mu_{S},
\end{aligned}
$$

cf. Evans and Gariepy [9, Theorem 1, p. 2]. Now Theorem 2.8(2), (3) together establish that

$$
\operatorname{meas}\left(\mathcal{P}_{2 m}\right) \rightarrow 0 \text { as } m \rightarrow \infty \text {. }
$$

Thus letting $m \rightarrow \infty$ in 4.12 yields

$$
\int_{\frac{1}{2} \Omega^{L}} \mu_{S}=\frac{1}{2} \int_{\Omega^{L}} \mu_{S}
$$

which with $\int_{\Omega^{L}} \mu_{S}=1$ proves Claim 1 .

Claim 2. Let $B^{\prime}=\frac{k}{2^{2 m}} \in \mathcal{B}^{\prime}$ and suppose that $x_{i}=B^{\prime}+\frac{x_{i}^{\prime}}{2^{2 m}}$ for $i=1,2$, with both $x_{i}^{\prime} \in \Omega^{L}$. Then

$$
\int_{x_{1}}^{x_{2}} \mu_{S}=\frac{1}{2^{2 m}}\left(\int_{x_{1}^{\prime}}^{x_{2}^{\prime}} \mu_{S}+\left(x_{2}^{\prime}-x_{1}^{\prime}\right)\right) .
$$

Since $B^{\prime}$ is a balanced dyadic rational, we may deduce (4.13) using the formula of Lemma 2.3 , in analogous fashion to 4.9 . This proves Claim 2.

Now we complete the proof. Claim 2 yields the formula

$$
\left|2^{2 m} \int_{x_{1}}^{x_{2}} \mu_{S}-\int_{x_{1}^{\prime}}^{x_{2}^{\prime}} \mu_{S}\right| \leq \operatorname{meas}\left(\left[x_{1}^{\prime}, x_{2}^{\prime}\right]\right) .
$$

For any $B^{\prime} \in \mathcal{B}^{\prime}$ we have

$$
\Omega^{L}\left(B^{\prime}\right):=\left\{x: x=B^{\prime}+\frac{x^{\prime}}{2^{2 m}} \text { with } x^{\prime} \in \frac{1}{2} \Omega^{L}\right\} .
$$


Now we may cover the set $\frac{1}{2} \Omega^{L}$ with $\frac{1}{2} \mathcal{P}_{2 n}$. We apply the approximation bound 4.14 summed up over all the intervals in $\mathcal{P}_{2 n}$, to obtain

$$
\left|2^{2 m} \int_{B^{\prime}+\frac{1}{2^{2 m}}\left(\frac{1}{2} \mathcal{P}_{2 n}\right)} \mu_{S}-\int_{\frac{1}{2} \mathcal{P}_{2 n}} \mu_{S}\right| \leq \frac{1}{2} \operatorname{meas}\left(\mathcal{P}_{2 n}\right) .
$$

Letting $n \rightarrow \infty$ we deduce, using meas $\left(\mathcal{P}_{2 n}\right) \rightarrow 0$, that

$$
2^{2 m} \int_{B^{\prime}+\frac{1}{2^{2 m}}\left(\frac{1}{2} \Omega^{L}\right)} \mu_{S}=\int_{\frac{1}{2} \Omega^{L}} \mu_{S}=\frac{1}{2} .
$$

This yields, since $\Omega^{L}\left(B^{\prime}\right):=B^{\prime}+\frac{1}{2^{2 m}}\left(\frac{1}{2} \Omega^{L}\right)$, that

$$
\int_{\Omega^{L}\left(B^{\prime}\right)} \mu_{S}=\frac{1}{2^{2 m+1}}
$$

as asserted.

\subsection{Singular measure mass of $\Omega_{\infty}^{L}$}

The calculations of the last section yield the following result.

Theorem 4.3. (Takagi singular measure: fine partition) Let $\mu_{S}$ denote the Takagi singular measure. The sets $\Omega_{\text {fin }}^{L}$ and $\Omega_{\infty}^{L}$ are Borel sets, hence measurable. We have

$$
\mu_{S}\left(\Omega_{f i n}^{L}\right)=1
$$

which shows that

$$
\mu_{S}\left(\Omega_{\infty}^{L}\right)=0
$$

Consequently the image of this set under the Takagi singular function $\tau^{S}$ satisfies

$$
\operatorname{meas}\left(\tau^{S}\left(\Omega_{\infty}^{L}\right)\right)=0
$$

where meas denotes Lebesgue measure.

Proof. Each set $\Omega^{L}\left(B^{\prime}\right)$ is closed, hence their disjoint union $\Omega_{\text {fin }}^{L}$ is a Borel set, hence is $\mu_{S^{-}}$ measurable. The set $\Omega^{L}$ is closed, hence $\Omega_{\infty}^{L}=\Omega^{L} \backslash \Omega_{\text {fin }}^{L}$ is also a Borel set, hence is $\mu_{S}$-measurable, and

$$
\mu_{S}\left(\Omega_{\infty}^{L}\right)=\mu_{S}\left(\Omega^{L}\right)-\mu_{S}\left(\Omega_{\text {fin }}^{L}\right)
$$

(In fact one can easily show that the closure of $\Omega_{\text {fin }}^{L}$ is $\Omega^{L}$.)

Since $\mu_{S}\left(\Omega^{L}\right)=1$ the theorem will follow on showing $\mu_{S}\left(\Omega_{\text {fin }}^{L}\right)=1$. We have

$$
\mu_{S}\left(\Omega_{f i n}^{L}\right)=\sum_{B \in \mathcal{B}^{\prime}} \mu_{S}\left(\Omega^{L}(B)\right)
$$

where $\mathcal{B}^{\prime}$ is the breakpoint set. Theorem 4.2 now gives $\mu_{S}\left(\Omega^{L}(B)\right)=\frac{1}{2^{2 m+1}}$, where $B=0 . b_{1} \cdots b_{2 m}=$ $\frac{k}{2^{2 m}}$, with $k$ odd. Recall from [19, Lemma 4.2] that the number of balanced dyadic rationals in $\Omega^{L}$ having the form $\frac{k}{2^{2 m}}$ for an odd $k$ is the $m$-th Catalan number $C_{m}=\frac{1}{m}\left(\begin{array}{c}2 m \\ m\end{array}\right)$. Here for $m=0$ we have $C_{0}=1$ corresponding to the element $B_{0}=0$. 
The Catalan numbers are well known to have the generating function

$$
\sum_{j=0}^{\infty} C_{m} z^{2 m}=\frac{1-\sqrt{1-4 z^{2}}}{2 z^{2}}
$$

In consequence, taking $z=\frac{1}{2}$, we obtain $\sum_{j=0}^{\infty} \frac{C_{m}}{2^{2 m}}=2$. Therefore we obtain, using Theorem 4.2 , that

$$
\mu_{S}\left(\Omega_{\text {fin }}^{L}\right)=\sum_{m=0}^{\infty} C_{m} \frac{1}{2^{2 m+1}}=\frac{1}{2}\left(\sum_{m=0}^{\infty} \frac{C_{m}}{2^{2 m}}\right)=1,
$$

which proves 4.15 . Now 4.16 follows, and 4.17 follows from Theorem 3.2 on taking $K=\Omega_{\infty}^{L}$ in (3.20).

\section{Cardinality of Global Level Sets}

In this section we prove Theorem 1.2, which states that for a full measure set of ordinates $y$ the level set $L(y)$ is a finite set, and that the expected number of elements in this set, with respect to Lebesgue measure on $0 \leq y \leq \frac{2}{3}$, is infinite.

We use the following result [19, Theorem 5.8] giving the expected number of local level sets in a uniformly chosen level $y$ in $\left[0, \frac{2}{3}\right]$.

Theorem 5.1. (Expected number of local level sets) For a full Lebesgue measure set of ordinate points $y \in\left[0, \frac{2}{3}\right]$ the number $N^{l o c}(y)$ of local level sets at level $y$ is finite. Furthermore

$$
\int_{0}^{\frac{2}{3}} N^{l o c}(y) d y=1
$$

That is, the expected number of local level sets on a randomly drawn ordinate level y is $\frac{3}{2}$.

Theorem 5.1 was proved using the coarea formula for functions of bounded variation applied to the flattened Takagi function $\tau^{L}$.

Proof of Theorem 1.2. (1) Let $\Gamma_{\infty}^{o r d}$ be the set of infinite levels, i.e.

$$
\Gamma_{\infty}^{o r d}:=\{y: L(y) \text { is an infinite set }\}
$$

To show a full measure set of ordinates have finite level sets, we show the contrapositive, that $\Gamma_{\infty}^{o r d}$ has Lebesgue measure 0 . We have

$$
\Gamma_{\infty}^{o r d} \subset \tau\left(\Omega_{\infty}^{L}\right) \bigcup \Lambda_{\infty}^{l o c},
$$

in which $\tau\left(\Omega_{\infty}^{L}\right):=\left\{y=\tau(x): x \in \Omega_{\infty}^{L}\right\}$ detects all levels that contain at least one infinite local level set, and

$$
\Lambda_{\infty}^{l o c}:=\{y: L(y) \text { contains infinitely many different local level sets }\} .
$$

Now [19, Theorem 7.2 (1)] shows that $\Lambda_{\infty}^{\text {loc }}$ has Lebesgue measure 0. Thus it suffices to prove that $\tau\left(\Omega_{\infty}^{L}\right)$ has Lebesgue measure 0. 
Recall that $\tau^{S}(x)=\tau(x)+x$, for $x \in \Omega^{L}$. Now consider $\tau^{S}(x)$ restricted to $x \in \Omega^{L}(B)$ for a particular $B \in \mathcal{B}^{\prime}$, the breakpoint set. We write $B=0 . b_{1} b_{2} \cdots b_{2 m}=\frac{k}{2^{2 m}}$ where $k$ is necessarily odd. Then $x \in \Omega^{L}(B)$ if and only if

$$
x=B+\frac{\frac{1}{2} x^{\prime}}{2^{2 m}}, \quad \text { with } \frac{1}{2} x^{\prime} \in \frac{1}{2} \Omega^{L} .
$$

Lemma 2.3 then gives

$$
\tau(x)=\tau(B)+\frac{1}{2^{2 m}} \tau\left(\frac{1}{2} x^{\prime}\right), \text { with } x^{\prime} \in \Omega^{L} .
$$

We recall from Lemma 2.1 that $2 \tau\left(\frac{1}{2}(x)\right)=\tau(x)+x$ if $x \in \Omega^{L}$, whence

$$
2^{2 m+1}(\tau(x)-\tau(B))=\tau\left(x^{\prime}\right)+x^{\prime}=\tau^{S}\left(x^{\prime}\right), \text { for } x^{\prime} \in \Omega^{L} .
$$

Now the linear map

$$
y \mapsto y^{\prime}:=2^{2 m+1}(y-\tau(B))
$$

sends the interval $\left[\tau(B), \tau(B)+\frac{1}{2^{2 m+1}}\right]$ onto $[0,1]$ and it follows from the above that it sends $\tau\left(\Omega^{L}(B)\right) \subset$ $\left[\tau(B), \tau(B)+\frac{1}{2^{2 m+1}}\right]$ onto the range $\tau^{S}\left(\Omega^{L}\right)=[0,1]$. We see from the linearity of this map that $\tau\left(\Omega^{L}(B)\right)=\left[\tau(B), \tau(B)+\frac{1}{2^{2 m+1}}\right]$,

$$
\text { meas }\left(\tau\left(\Omega^{L}(B)\right)\right)=\frac{1}{2^{2 m+1}} .
$$

Adding up these contributions, the summation in Theorem 4.3 gives that the total Lebesgue measure in $y \in\left[0, \frac{2}{3}\right]$ covered by images of these sets (counting overlaps with multiplicity) is

$$
\sum_{B \in \mathcal{B}^{\prime}} \operatorname{meas}\left(\tau\left(\Omega^{L}(B)\right)\right)=\sum_{B \in \mathcal{B}^{\prime}} \mu_{S}\left(\Omega^{L}(B)\right)=1 .
$$

(The images have some overlap, allowing their total measure to exceed the length of the interval $\left[0, \frac{2}{3}\right]$.) Viewing these points $x \in \Omega^{L}(B)$ as labelling left endpoints of local level sets, this says that a lower bound of the total number of local level set endpoints Lebesgue-integrated over $0 \leq y \leq \frac{2}{3}$, counted with multiplicity, is 1 . Here we did not count any local level set endpoints in $\tau\left(\Omega_{\infty}^{L}\right):=$ $\tau\left(\Omega^{L} \backslash \Omega_{\text {fin }}^{L}\right)$, coming from the image of $\Omega_{\infty}^{L}$, since each $\Omega^{L}(B)$ is disjoint from $\Omega_{\infty}^{L}$. Theorem 5.1 now gives

$$
\int_{0}^{\frac{2}{3}} N^{l o c}(y) d y=1
$$

where $N^{l o c}(y)$ counts the number of all local level set endpoints. We have already accounted for the full mass of this integral above, and any omitted contribution to $N^{l o c}(y)$ coming from $\tau\left(\Omega_{\infty}^{L}\right):=$ $\tau\left(\Omega^{L} \backslash \Omega_{\text {fin }}^{L}\right)$ necessarily contributes an additional nonnegative amount. Thus we may conclude that

$$
\operatorname{meas}\left(\tau\left(\Omega_{\infty}^{L}\right)\right)=0
$$

as asserted.

(2) We aim to show that the expected size of a global level set is infinite, i.e. to show that

$$
\int_{0}^{\frac{2}{3}}|L(y)| d y=+\infty
$$


where $|L(y)|$ counts the number of elements in $L(y)$. By the discussion above we have

$$
\int_{0}^{\frac{2}{3}}|L(y)| d y=\int_{0}^{1}|L(\tau(x))| \mu_{S}(x) \geq \sum_{B \in \mathcal{B}^{\prime}} \frac{1}{2^{2 m+1}} 2^{r(B)},
$$

in which

$$
r(B):=\left|\left\{1 \leq j<\infty: N_{j}(B)=0\right\}\right| .
$$

Here each $r(B)$ is finite and bounded above by $m$ if $B=0 . b_{1} b_{2} \cdots b_{2 m}$. We rewrite this as

$$
\int_{0}^{\frac{2}{3}}|L(y)| d y=\sum_{m=0}^{\infty} \frac{L_{m}}{2^{2 m+1}}
$$

in which

$$
L_{m}:=\sum_{B \in \mathcal{B}^{\prime},|B|=2 m} 2^{r(B)} .
$$

Now we observe that $L_{m}$, the total number of binary sequences of length $2 m$ having $N_{2 m}(B)=0$, has a combinatorial interpretation as counting the number the two-dimensional lattice paths of length $2 m$ starting at the origin $(0,0)$, taking steps either $(1,1)$ or $(1,-1)$, and ending at $(2 m, 0)$. Indeed these paths groups into collections of paths of size $2^{r}$ under the "flipping" (reflection) operation, with each group containing exactly one path in $\mathcal{B}^{\prime}$. (See the discussion and proof in Feller [12, Theorem 4, p. 90] and also [19, Lemma 4.2].) It follows that

$$
L_{m}=\left(\begin{array}{c}
2 m \\
m
\end{array}\right) \sim \frac{1}{\sqrt{\pi m}} 2^{2 m} .
$$

Thus the terms in the series on the right side of 5.6 decay like $\Omega\left(\frac{1}{\sqrt{m}}\right)$, so the series 5.6 diverges, giving the result.

\section{Level Sets of Positive Hausdorff Dimension: Abscissa View}

We study level sets having positive Hausdorff dimension. In the paper [19, Sect. 3.3]) we classified those local level sets containing a rational number $x$ that are of positive Hausdorff dimension: this gives some explicitly determinable rational ordinates $y$ having this property. Here we show that the set $\Gamma_{H}^{L}$ of abscissa points in $\Omega^{L}$ that give local level sets having positive Hausdorff dimension has full Hausdorff dimension 1.

Theorem 6.1. (Local level sets of positive Hausdorff dimension) Let $\Lambda_{H}^{L}$ denote the set of $x \in \Omega^{L}$ such that the Hausdorff dimension of $L_{x}^{\text {loc }}$ is positive, i.e.

$$
\Lambda_{H}^{L}:=\left\{x \in \Omega^{L}: \operatorname{dim}_{H}\left(L_{x}^{l o c}\right)>0\right\} .
$$

This set has full Hausdorff dimension, i.e.

$$
\operatorname{dim}_{H}\left(\Lambda_{H}^{L}\right)=1
$$

In particular, the deficient digit set $\Omega^{L}$ has Hausdorff dimension 1. 
Proof. It clearly suffices to prove the first assertion. For integer $r \geq 1$ let $\Gamma_{2 r}$ consist of all abscissas $x \in[0,1]$ that satisfy:

(i) $D_{j}(x)>0$ for $j \not \equiv 0(\bmod 2 r)$.

(ii) $D_{2 k r}(x)=0$ for $k=1,2,3, \ldots$

These conditions are equivalent to requiring $\Gamma_{2 r} \subset \Omega^{L}$, and that all $x \in \Gamma_{2 r}$ have the same balance-set $Z(x):=\left\{k: D_{k}(x)=0\right\}=2 r \mathbb{N}$.

Claim 1. All members $x \in \Gamma_{2 r}$ have local level sets of positive Hausdorff dimension, satisfying

$$
\operatorname{dim}_{H}\left(L_{x}^{l o c}\right) \geq \frac{\log 2}{\log \left(2^{2 r}\right)}=\frac{1}{2 r} .
$$

Claim 1 will follow from the spacing of the balance points being an arithmetic progression. This makes each local level set $L_{x}^{l o c}$ a Cantor-like set, which has a standard tree construction covered by $2^{k}$ intervals of length $2^{-2 r k}$, so that $\operatorname{dim}_{H}\left(L_{x}^{l o c}\right)=\frac{1}{2 r}$. Note that the particular subintervals are chosen differently at each step, so that this is generally not a self-similar construction, but the Hausdorff dimension lower bound proof for the Cantor set given in Falconer [11, Sect. 2.3] remains valid here, establishing Claim 1.

Claim 1 shows that $\Gamma_{2 r} \subset \Lambda_{H}^{L}$, so that

$$
\bigcup_{r=1}^{\infty} \Gamma_{2 r} \subset \Lambda_{H}^{L} .
$$

To complete the proof it suffices to show the sets $\Gamma_{2 r}$ each have positive Hausdorff dimension, which approaches 1 as $r \rightarrow \infty$.

Claim 2. For all large enough $r$, the set $\Gamma_{2 r}$ has Hausdorff dimension greater than $1-\frac{2 \log r}{r}$.

Claim 2 follows by observing that $\Gamma_{2 r}$ is itself a self-similar Cantor set in which each block of $2 r$ symbols is drawn from the set

$$
X_{2 r}:=\left\{x=\frac{m}{2^{2 r}}=0 . b_{1} b_{2} \ldots b_{2 r}: \quad D_{j}(x)>0 \text { for } 1 \leq j<2 r, D_{2 r}(x)=0\right\},
$$

whose Hausdorff dimension is computable by the method of Falconer [11, Sect. 2.3]. It is well known that

$$
\left|X_{2 r}\right|=C_{r}=\frac{1}{r+1}\left(\begin{array}{c}
2 r \\
r
\end{array}\right)
$$

is a Catalan number. Thus we obtain

$$
\operatorname{dim}_{H}\left(\Gamma_{2 r}\right)=\frac{\log C_{r}}{\log 2^{2 r}}=\frac{\log C_{r}}{2 r \log 2} .
$$

However it is well known that $C_{r}=\frac{2^{2 r}}{\pi r^{\frac{3}{2}}}(1+o(1))$, as the integer $r \rightarrow \infty$. We conclude that for large enough $r$ there holds

$$
\operatorname{dim}_{H}\left(\Gamma_{2 r}\right)>1-\frac{2 \log r}{r} .
$$

Claim 2 now follows, and the theorem is proved.

Note that Claims 1 and 2 together imply that the local level set dimension spectrum function $f_{\tau}^{*}(\alpha)$ defined in Section 1.2 satisfies, $f_{\tau}^{*}\left(\frac{1}{2 r}\right)>1-\frac{2 \log r}{r}$ for all sufficiently large integers $r$. 
Proof of Theorem 1.4. We have $\Lambda_{H}^{L} \subset \Gamma_{H}^{a b s}$. Theorem 6.1 then gives $\operatorname{dim}_{H}\left(\Gamma_{H}^{a b s}\right) \geq \operatorname{dim}_{H}\left(\Lambda_{H}^{L}\right)=$ 1. yielding $\operatorname{dim}_{H}\left(\Gamma_{H}^{a b s}\right)=1$.

\section{Level Sets of Positive Hausdorff Dimension: Ordinate View}

Our object is to prove Theorem 1.5, which asserts that the set of ordinate levels $y$ having $\operatorname{dim}_{H}(L(y))>$ 0 has Hausdorff dimension 1. We use the result on abscissas proved in the last section (Theorem 6.1), together with a property showing that the Takagi function restricted to certain small domains in $[0,1]$ is quite well behaved, i.e. it is bi-Lipschitz map. This allows the transfer of Hausdorff dimension lower bounds from the abscissa case treated in Sect. 6.

\subsection{Bi-Lipschitz property of Takagi function on $\Gamma_{2 r}$}

The following proof of the bi-Lipschitz property incorporates a substantial simplification of the authors' original argument, due to P. Allaart.

Theorem 7.1. Let $Z(x)=\left\{j: D_{j}(x)=0\right\}$ be the balance-set of $x \in[0,1]$. For $r \geq 1$ the Takagi function $\tau(x)$ restricted to the (compact) domain

$$
\Gamma_{2 r}:=\left\{x \in \Omega^{L}: Z(x)=2 r \mathbb{N}\right\}
$$

is strictly increasing and is a bi-Lipschitz map.

Proof. We will show that if $x_{1}<x_{2}$ are both in $\Gamma_{2 r}$ then we have the bi-Lipschitz estimates

$$
2^{2 r}\left(x_{2}-x_{1}\right) \geq \tau\left(x_{2}\right)-\tau\left(x_{1}\right) \geq \frac{1}{2^{2 r}}\left(x_{2}-x_{1}\right) .
$$

The lower bound shows that the Takagi function restricted to $\Gamma_{2 r}$ is strictly increasing.

To prove (7.1), we first reduce to the case that $x_{1}$ and $x_{2}$ have binary expansions that disagree somewhere in the first $2 r$ digits. If they disagree first between the $2 k r+1$ and $2(k+1) r$ digits, then we can write $x_{i}=x_{0}+\frac{w_{i}}{2^{2 k r}}$ with $0 \leq w_{i} \leq 1$, where $x_{0}=0 . b_{1} b_{2} \cdots b_{2 k r}$ is the initial block of $2 k r$ digits where they agree, and $w_{1}<w_{2}$. Note that $w_{1}, w_{2} \in \Lambda_{2 r}$ by the self-similar definition of $\Lambda_{2 r}$, and $w_{1}, w_{2}$ disagree somewhere in their first $2 r$ digits. Now

$$
x_{2}-x_{1}=\frac{1}{2^{2 k r}}\left(w_{2}-w_{1}\right)
$$

and, since $D_{2 k r}\left(x_{1}\right)=D_{2 k r}\left(x_{2}\right)=0$, Lemma 2.3 gives

$$
\tau\left(x_{2}\right)-\tau\left(x_{1}\right)=\frac{1}{2^{2 k r}}\left(\tau\left(w_{2}\right)-\tau\left(w_{1}\right)\right) .
$$

Thus it suffices to prove (7.1) for $w_{1}$ and $w_{2}$.

By definition all $x=0 . b_{1} b_{2} \ldots \in \Lambda_{2 r}$ have $D_{m}(x)>0$ for all $m \neq 2 k r$, while $D_{2 k r}(x)=0$ for all $k \geq 1$. This forces $b_{2 k r+1}=b_{2 k r+2}=0$ and $b_{2(k+1) r-1}=b_{2(k+1) r-2}=1$, for all $k \geq 0$. We now suppose $x_{1}=0 . b_{1} b_{2} \ldots$ and $x_{2}=0 . b_{1}^{\prime} b_{2}^{\prime} \ldots$ disagree somewhere in their first $2 r$ digits, say at the $n$-th digit, where $3 \leq n \leq 2 r-2$. Now define the dyadic rationals

$$
\tilde{x}_{1}:=0 . b_{1} b_{2} \ldots b_{2 r-2} 10^{\infty}=\frac{k_{1}}{2^{2 r-1}},
$$




$$
\tilde{x}_{2}:=0 . b_{1}^{\prime} b_{2}^{\prime} \ldots b_{2 r-2}^{\prime} 10^{\infty}=\frac{k_{2}}{2^{2 r-1}},
$$

which truncate the $x_{i}$ at their $(2 r-1)$-st digits, and the dyadic rationals

$$
\begin{aligned}
& \bar{x}_{1}:=0 . b_{1} b_{2} \ldots b_{2 r-2} 110^{\infty}=\frac{2 k_{1}+1}{2^{2 r}}, \\
& \bar{x}_{2}:=0 . b_{1}^{\prime} b_{2}^{\prime} \ldots b_{2 r-2}^{\prime} 110^{\infty}=\frac{2 k_{2}+1}{2^{2 r}},
\end{aligned}
$$

which truncate the $x_{i}$ at their $2 r$-th digits. We note that $D_{2 r-1}\left(\tilde{x}_{i}\right)=1$ and $D_{2 r}\left(\bar{x}_{i}\right)=0$ for $i=1,2$. We can now write

$$
x_{i}=\bar{x}_{i}+\frac{x_{i}[2 r]}{2^{2 r}}, \quad \text { for } i=1,2,
$$

in which $x_{1}[2 r]=0 . b_{2 r+1} b_{2 r+2} \cdots$ and similarly for $x_{2}[2 r]$. Since each $x_{i}[2 r]=0.00 \ldots$ and each $\bar{x}_{i}=0.00 \ldots$ we deduce $\left|x_{2}[2 r]-x_{1}[2 r]\right| \leq \frac{1}{4}$, and

$$
\frac{1}{4} \geq x_{2}-x_{1} \geq\left(\bar{x}_{2}-\overline{x_{1}}\right)-\frac{1}{4} \cdot \frac{1}{2^{2 r}} .
$$

Now $\bar{x}_{2}-\bar{x}_{1} \geq \frac{1}{2^{2 r-1}}$ so we obtain

$$
\frac{1}{4} \geq x_{2}-x_{1} \geq \frac{7}{8} \cdot \frac{1}{2^{2 r-1}} .
$$

Next we estimate the difference between $\tau\left(x_{2}\right)$ and $\tau\left(x_{1}\right)$. For the lower bound, we will relate it to the difference between $\tau\left(\bar{x}_{2}\right)$ and $\tau\left(\bar{x}_{1}\right)$. Using Lemma 2.3 and $D_{2 r}\left(\bar{x}_{i}\right)=0$ for $i=1,2$, we obtain

$$
\tau\left(x_{i}\right)=\tau\left(\bar{x}_{i}\right)+\frac{\tau\left(x_{i}[2 r]\right)}{2^{2 r}}, \text { for } i=1,2 .
$$

This yields, using $0 \leq \tau\left(x_{i}[2 r]\right) \leq \frac{2}{3}$,

$$
\tau\left(x_{2}\right)-\tau\left(x_{1}\right) \geq\left(\tau\left(\bar{x}_{2}\right)-\tau\left(\bar{x}_{1}\right)\right)-\frac{2}{3} \cdot \frac{1}{2^{2 r}} .
$$

To estimate the right side, we will relate these quantities to $\tau\left(\tilde{x}_{i}\right)$. Using $\bar{x}_{i}=\tilde{x}_{i}+\frac{1}{2^{2 r}}$, a calculation using Lemma 2.3 gives

$$
\tau\left(\bar{x}_{i}\right)=\tau\left(\tilde{x}_{i}\right)+\frac{1}{2^{2 r-1}}, \text { for } i=1,2
$$

Thus (7.3) becomes

$$
\tau\left(x_{2}\right)-\tau\left(x_{1}\right) \geq\left(\tau\left(\tilde{x}_{2}\right)-\tau\left(\tilde{x}_{1}\right)\right)-\frac{2}{3} \cdot \frac{1}{2^{2 r}} .
$$

Now comes the key observation. Both $\tilde{x}_{1}, \tilde{x}_{2} \in \frac{1}{2} \Omega^{L}$, since $D_{m}\left(\tilde{x}_{i}\right) \geq 1$ for $1 \leq i \leq 2 r-1$. By hypothesis $x_{1}$ and $x_{2}$ disagree at the $n$-th digit, with $n \leq 2 r-2$, at which $b_{n}=0, b_{n}^{\prime}=1$, and we define the intermediate dyadic rational

$$
x_{0}:=0 . b_{1}^{\prime} b_{2}^{\prime} \cdots b_{n}^{\prime} 0^{\infty}=\frac{k_{0}}{2^{n}} .
$$

We have $x_{0} \in \frac{1}{2} \Omega^{L}$ because its binary expansion is a prefix of that of $x_{2}$. Furthermore $x_{0}<x_{2}$ because $x_{0}$ and $x_{2}$ disagree at the $(2 r-1)$-st digit. Thus $x_{1}<x_{0}<x_{2}$ and we may now apply Theorem 2.9(2) 
to conclude the strict inequality $\tau\left(\tilde{x}_{1}\right)<\tau\left(\tilde{x}_{2}\right)$. But $\tau\left(\tilde{x}_{i}\right)(i=1,2)$ are both dyadic rationals with denominator at most $2^{2 r-1}$, so we deduce that

$$
\tau\left(\tilde{x}_{2}\right)-\tau\left(\tilde{x}_{1}\right) \geq \frac{1}{2^{2 r-1}} .
$$

Substituting this inequality into (7.4) yields the lower bound

$$
\tau\left(x_{2}\right)-\tau\left(x_{1}\right) \geq \frac{4}{3} \cdot \frac{1}{2^{2 r}} .
$$

This proves the function $\tau(x)$ is strictly increasing on $\Gamma_{2 r}$, and also gives the desired bi-Lipschitz estimate.

Combining 7.5 with the trivial upper bound estimate $\tau\left(x_{2}\right)-\tau\left(x_{1}\right) \leq \frac{2}{3}$, and with 7.2 yields

$$
2^{2 r-1}\left(x_{2}-x_{1}\right) \geq \tau\left(x_{2}\right)-\tau\left(x_{1}\right) \geq \frac{1}{2^{2 r-2}}\left(x_{2}-x_{1}\right)
$$

This implies (7.1).

Remark. The Takagi function $\tau(x)$ is not a Lipschitz map on its full domain $[0,1]$, nor is it a Lipschitz function even when restricted to the domain $\Omega^{L}$. This is because it has arbitrarily steep slopes on $\Omega^{L}$, as is implicit in the singular function property.

\subsection{Hausdorff dimension of $\Gamma_{H}^{o r d}$}

To conclude the paper we prove Theorem 1.5 .

\section{Proof of Theorem 1.5. Let}

$$
\Gamma_{H}^{o r d}:=\left\{y: 0 \leq y \leq \frac{2}{3} \text { with } \operatorname{dim}_{H} L(y)>0\right\} .
$$

It is well known that bi-Lipschitz maps preserve Hausdorff dimension. By Theorem 7.1 the bi-Lipschitz property holds for the Takagi function $\tau$ restricted to the compact domain $\Gamma_{2 r}$. The range of this map is

$$
\tilde{\Gamma}_{2 r}:=\left\{y: y=\tau(x), x \in \Gamma_{2 r}\right\}
$$

which therefore satisfies

$$
\operatorname{dim}_{H}\left(\tilde{\Gamma}_{2 r}\right)=\operatorname{dim}_{H}\left(\Gamma_{2 r}\right) \geq 1-\frac{2 \log r}{2 r} .
$$

for large enough $r$, as shown in Claim 2 of the proof of Theorem6.1.

Next, Claim 1 of that proof shows that every level $y \in \Gamma_{2 r}$ has

$$
\operatorname{dim}_{H}(L(y)) \geq \operatorname{dim}_{H}\left(L_{x}^{l o c}\right) \geq \frac{1}{2 r} .
$$

Combining these inequalities shows that the Takagi dimension spectrum function satisfies

$$
f_{\tau}\left(\frac{1}{2 r}\right) \geq 1-\frac{2 \log r}{2 r}
$$

for all sufficiently large $r$. Thus we have $\tilde{\Gamma}_{2 r} \subset \Gamma_{H}^{o r d}$. and

$$
\operatorname{dim}_{H}\left(\Gamma_{H}^{o r d}\right) \geq 1-\frac{2 \log r}{r}
$$

Letting $r \rightarrow \infty$ gives $\operatorname{dim}_{H}\left(\Gamma_{H}^{o r d}\right) \geq 1$, establishing the result. 


\section{References}

[1] P. C. Allaart, How large are the level sets of the Takagi function? eprint: arXiv:1102.1616 v1, v1, 8 Feb 2011.

[2] P. C. Allaart, The finite cardinalites of the level sets of the Takagi function, eprint: arXiv:1107.0712, v1, 4 Jul 2011.

[3] P. C. Allaart and K. Kawamura, Extreme values of some continuous nowhere differentiable functions, Math. Proc. Camb. Phil. Soc. 140 (2006), No. 2, 269-295.

[4] P. C. Allaart and K. Kawamura, The improper infinite derivatives of Takagi's nowheredifferentiable function, J. Math. Anal. Appl. 372 (2010), 656-665.

[5] E. de Amo, I. Bhouri, M. Díaz Carrillo, and J. Fernándex-Sánchez, Hausdorff dimension of Takagi's function level sets, Nonlinear Analysis 74 (2011), No. 15, 5081-5087.

[6] Y. Baba, On maxima of Takagi-van der Waerden functions, Proceedings of the American Mathematical Society, 91 (1984), no. 3, 373-376.

[7] Z. Buczolich, Irregular 1-sets on the graphs of continuous functions, Acta Math. Hungar. 121 (2008), 371-393.

[8] H. Delange, Sur la fonction sommatoire de la fonction "somme des chiffres", Enseign. Math. 21 (1975), 31-47.

[9] L. C. Evans and R. G. Gariepy, Measure Theory and Fine Properties of Functions, CRC Press: Boca Raton 1992.

[10] K. Falconer, The Geometry of Fractal Sets, Cambridge University Press: Cambridge 1985.

[11] K. Falconer, Fractal Geometry: Mathematical Foundations and Applications, Second Edition, John Wiley \& Sons: New York 2003.

[12] W. Feller, An Introduction to Probability Theory and its Applications, Vol I. Third Edition, John Wiley \& Sons, New York 1968.

[13] P. R. Halmos, Measure Theory, Van Nostrand: Princeton NJ 1950.

[14] M. Hata and M. Yamaguti, The Takagi function and its generalization, Japan J. Appl. Math. 1 (1984), 183-199.

[15] S. Jaffard, Multifractal formalism for functions part I: results valid for all functions, SIAM J. Math. Anal. 28 (1997), No. 4, 944-970.

[16] S. Jaffard, Multifractal formalism for functions part II: Self-similar functions, SIAM J. Math. Anal. 28 (1997), No. 4, 971-998.

[17] D. E. Knuth, The Art of Computer Programming, Vol. 4, Fascicle 3. Generating all Combinations and Partitions, Addison-Wesley: Upper Saddle River, NJ 2005.

[18] J. C. Lagarias, The Takagi function and its properties, paper in preparation.

[19] J. C. Lagarias and Z. Maddock, Level sets of the Takagi function: Local level sets, preprint. 
[20] Z. Maddock, Level sets of the Takagi function: Hausdorff dimension, Monatshefte für Math. 160 (2010), 167-186.

[21] R. D. Mauldin and S. C. Williams, On the Hausdorff dimension of some graphs, Trans. Amer. Math. Soc. 298, No. 2 (1986), 793-803.

[22] L. Mirsky, A theorem on representation of integers in the scale of $r$, Scripta Math. 15 (1949), $11-12$

[23] T. Takagi, A simple example of the continuous function without derivative, Proceedings of the Physico-Mathematical Society of Japan, ser II, Vol 1. 1903, pp 176-177. [Also: Collected Papers of Teiji Takagi (S. Iyanaga, Ed), Springer Verlag, New York 1990].

[24] J. R. Trollope, An explicit expression for binary digital sums, Math. Mag. 41 (1968), 21-25.

[25] M. Yamaguti, M. Hata and J. Kigami, Mathematics of Fractals, Translations of Mathematical Monographs, No. 167, American Mathematical Society: Providence, RI 1997. (Translation from Japanese of 1993 original.)

Jeffrey C. Lagarias

Dept. of Mathematics

The University of Michigan

Ann Arbor, MI 48109-1043

email: lagarias@umich.edu

Zachary Maddock

Dept. of Mathematics

Columbia University

New York, NY 10027

email: maddockz@math. columbia.edu 